\title{
Wild Bee Toxicity Data for Pesticide Risk Assessments
}

\author{
Kathleen A Lewis* (D) and John Tzilivakis $(\mathbb{D}$ \\ Agriculture \& the Environment Research Unit, University of Hertfordshire, Hatfield AL10 9AB, UK \\ * Correspondence: k.a.lewis@herts.ac.uk; Tel.: +44-1707-284582
}

Received: 20 June 2019; Accepted: 9 July 2019; Published: 11 July 2019

\begin{abstract}
Pollination services are vital for agriculture, food security and biodiversity. Although many insect species provide pollination services, honeybees are thought to be the major provider of this service to agriculture. However, the importance of wild bees in this respect should not be overlooked. Whilst regulatory risk assessment processes have, for a long time, included that for pollinators, using honeybees (Apis mellifera) as a protective surrogate, there are concerns that this approach may not be sufficiently adequate particularly because of global declines in pollinating insects. Consequently, risk assessments are now being expanded to include wild bee species such as bumblebees (Bombus spp.) and solitary bees (Osmia spp.). However, toxicity data for these species is scarce and are absent from the main pesticide reference resources. The aim of the study described here was to collate data relating to the acute toxicity of pesticides to wild bee species (both topical and dietary exposure) from published regulatory documents and peer reviewed literature, and to incorporate this into one of the main online resources for pesticide risk assessment data: The Pesticide Properties Database, thus ensuring that the data is maintained and continuously kept up to date. The outcome of this study is a dataset collated from 316 regulatory and peer reviewed articles that contains 178 records covering 120 different pesticides and their variants which includes 142 records for bumblebees and a further 115 records for other wild bee species.
\end{abstract}

Keywords: bee toxicity; risk assessment; environmental toxicology

\section{Summary}

Pollination services are vital for agriculture, food security and biodiversity. It has been estimated that at least $84 \%$ of all EU crops ( $75 \%$ of the most important) and $80 \%$ of wildflowers rely on insect pollination [1,2]. Many birds and small mammals rely on seeds, fruits and berries from plants that are pollinated by bees. There is no doubt that without pollination agricultural production would suffer considerably and our countryside would be lacking the habitats and food sources wild plants provide and upon which fauna depend.

Although many insect species provide pollination services, honeybees are thought to be the major provider of this service to agriculture. Research has suggested that around $80 \%$ of agricultural pollination services world-wide are provided by the domesticated European Honeybee (Apis mellifera) [3]. However, the importance of wild bees, especially bumblebees (Bombus spp.), should not be overlooked [4]. Bumblebees are more specialist pollinators than honeybees and are particularly important for greenhouse crops and wild plants. They are highly efficient at the pollination process due to their body size enabling them to carry large pollen loads, their variable tongue length, and their longer season, being one of the first bees to emerge after winter. In addition, bumblebees (and some other wild bee species) use a special technique known as 'buzz pollination' or sonication whereby flight muscles are contracted to produce strong vibrations that effectively shake the pollen loose from the plant $[4,5]$. 
Estimating the economic value of pollination services is challenging. However, in 2005 it was calculated to be around $£ 170$ billion ( $\$ 215$ billion) per year for global food production and $£ 12$ billion ( $\$ 15$ billion) per year for the EU [6]. Whilst the Buglife charity [7] stated it was $£ 12.6$ billion for the $\mathrm{EU}$ ( $\$ 16$ billion). For the UK alone the 2007 value has been given as $£ 430$ million ( $\$ 550$ million) [8,9]. These figures do not take into account the income and other societal benefits, such as employment, derived from industries and other commercial enterprises based on bee products such as honey, royal jelly, propolis and bees wax. Despite the obvious value to society we have in the past tended to take pollination services for granted and, perhaps, not given it as much protection as we should have. Now many of our insect pollinators are in decline, threatened by numerous environmental pressures including climate change, loss and fragmentation of habitats particularly flower-rich semi-natural areas, disease and parasites, invasive species, agricultural intensification and pesticide use [10-12].

Regulatory risk assessments for pesticides in most of the developed world have, for a long time, included evaluation of the risk to pollinators. Many, including the European Food Safety Authority (EFSA), Health Canada Pest Management Regulatory Authority (HCPRA) and the US Environmental Protection Authority (US EPA) use a framework encompassing a tiered approach where the findings from one tier may trigger the need for a more rigorous assessment offered by the next higher tier $[13,14]$. This pragmatic approach means that each tier offers a more comprehensive risk assessment with less uncertainty than the previous tier but will also often be more time consuming to undertake and more expensive. Generally, the bottom tier uses laboratory generated data whilst higher tiers will be more dependent on field studies and consider risks to whole populations rather than just individuals. In the case of pollinators, the first tier is usually the determination of an exposure-toxicity ratio (ETR-for dietary exposure) or a simple hazard quotient (HQ — for contact exposure) determined by dividing the predicted environmental concentration by a measure of toxicity, usually the acute median lethal dose (worst case 24,48 or $72 \mathrm{~h} \mathrm{LD}_{50}$ ) defined as the lethal dose for $50 \%$ of the tested population. This value is then compared to a threshold value (also known as trigger value or 'level of concern' value) used to highlight a potential concern and so initiate higher tier assessments. For higher tier assessments the aim is to demonstrate, via field studies under conditions similar to that of the proposed uses, that there are no unacceptable effects on honeybee larvae, honeybee behavior, or survival and development of bee colonies following application of the pesticide $[13,14]$.

Up until relatively recently honeybees were used as a protective surrogate for all bees in most regulatory risk assessments, based on the premise that protecting honeybees also protected bumblebees and solitary bees. However, recent research suggested that this may not always be a sound approach for several reasons. Firstly, Apis and non-Apis bees may vary in their susceptibility to pesticides and pesticides exposure opportunities may be different due to variations in feeding and breeding behavior, and chosen habitats [15-19]. There is also an issue on the metrics used for toxicity data. Each datum is usually reported as $\mu \mathrm{g}$ per bee but different bee species have very different bodyweights ranging from around $300 \mathrm{mg}$ for Bombus terrestris, $100 \mathrm{mg}$ for Apis mellifera and just $8 \mathrm{mg}$ for Nomia melanderi. Normalizing to account for weight differences has been shown not to be reliable [12,20]. These types of issues coupled with growing concerns over declines in pollinator populations have led to a number of improvements in regulator risk assessments $[10,13]$. One such improvement proposed by several regulatory authorities, including the EFSA [13] and the US EPA [14] is the inclusion into the risk evaluation process of one or more wild bee species. EFSA has proposed the inclusion of the bumblebee (Bombus terrestris) and the red mason bee (Osmia bicornis) whilst the US EPA suggest the bumblebee, the blue orchard bee (Osmia lignaria) and the alfalfa leafcutting bee (Megachile rotundata). However, whilst data for these species tend to be more frequently included in data packages reported and used to support the regulatory processes this only affects new pesticides or those subject to re-evaluation; it is not retrospective. In addition, the inclusion of data for non-Apis bees within these data packages is not mandatory and honeybee data still tends to be used as a surrogate.

Although the need for this type of data for regulatory risk assessments has been apparent for many years, available data tends not to be included in any of the main reference sources [21-24]. There 
have been attempts to collate it in the past but this work has not been for the sole purpose of creating a managed dataset. For example, Arena and Sgolastra [16] created a dataset for a meta-analysis to compare the sensitivity of various bee species to pesticides, and Sanchez-Bayo and Goka [25] also collated a similar dataset to undertake a risk assessment. In both these cases, whilst the datasets were published as supplementary information accompanying a journal article, they have not been included in any of the main public pesticide reference sources and they have not been updated or managed in any way that is evident. So, although there are a number of published studies available, and the number is increasing year on year, albeit relatively slowly, identifying and extracting useful data is a time consuming exercise because it is scattered throughout scientific literature as well as in monograph-type regulatory dossiers. This will remain a problem until it is routinely included into one or more of the major reference sources.

The Pesticide Properties Database (PPDB) is described in detail by Lewis and Tzilivakis [24]. It is an online database that holds a comprehensive range of data routinely used in pesticide risk assessments including those relating to pesticide chemistry, various parameters that describe the fate and transport of pesticides in the environment, large sections relating to human health and ecotoxicology as well as information on regulatory approvals and commercial products. The resource is used world-wide, with around 3000-5000 page loads of information retrieved daily. It is externally peer reviewed, endorsed by the International Union of Pure and Applied Chemistry (IUPAC) and promoted by several major organizations including the Food and Agriculture Organisation (FAO) and several environmental protection authorities including those from Denmark, Germany and Slovenia. The database is updated and managed continuously according to prescribed protocols that describe the preferred metrics, experimental conditions (e.g., temperature, pressure) and the representative species. There is no change for accessing the online system. It is also available, under license, in various formats including as MS Access or MS Excel files. These formats allow the database to be used offline or linked directly to mathematical models and decision support systems [24].

Prior to the work reported here the PPDB did not contain ecotoxicological data relating to wild bees, although honeybee data, both topical and dietary, have been available for many years. Consequently, the aim of this study was to create a dataset from regulatory dossiers and peer reviewed literature and use this to supplement existing ecotoxicological data currently in the PPDB. The data would then be subject to the PPDBs continuous updating and management program. The dataset presented in this manuscript is available as a Supplementary datafile and it has now been incorporated into the PPDB. New data will be added as and when it is identified. Thus, the data are now easily accessible by the PPDB's global user base for use in risk assessments.

\section{Data Description}

\subsection{Data Structure}

The dataset as presented herein has a very simple structure (see Table S1 of the Supplementary Material. It consists of a single MS Excel file comprised of three datasheets. Sheet 1 contains the bee/pesticide toxicity data and is comprised of 20 parameters as shown in Table 1 . These includes three parameters to aid correct chemical identification (common name, IUPAC chemical name and CAS RN) and an additional parameter, the PPDB code, which is a unique identifier for the PPDB. The use of this code is described in Section 4. The final parameter in this first section is used to define the mode of exposure, either topical or dietary. There are then three groups of three parameters each defining the toxicity, the species the data refers to and the bibliographical reference number for (i) Apis mellifera, (ii) Bombus spp., (iii) Osmia spp. The final two sets of three parameters are used for other species of bees. Data for Apis mellifera in this dataset has been included to ease comparisons and this has all been extracted directly from the PPDB. 
Table 1. Description of the dataset variables.

\begin{tabular}{|c|c|}
\hline Parameter & Description \\
\hline Pesticide common name & $\begin{array}{l}\text { The name by which the pesticide active substance is commonly known. } \\
\text { Data in this column are listed alphabetically. }\end{array}$ \\
\hline Pesticide chemical name & $\begin{array}{l}\text { The pesticide chemical nomenclature according to the naming rules of the } \\
\text { International Union of Pure and Applied Chemistry (IUPAC). }\end{array}$ \\
\hline CAS Registry Number & $\begin{array}{c}\text { The Chemical Abstract Services' unique identifying number assigned to } \\
\text { the pesticide. }\end{array}$ \\
\hline $\begin{array}{l}\text { PPDB Code } \\
\text { Exposure type }\end{array}$ & $\begin{array}{l}\text { Unique identifier linking the record to the PPDB (see User Notes below). } \\
\text { Exposure route of the bee to the pesticide: topical or dietary }\end{array}$ \\
\hline $\mathrm{LD}_{50}$ Apis spp. $\left(\mu \mathrm{g}\right.$ bee $\left.^{-1}\right)$ & $\begin{array}{l}\text { Lethal Dose, } 50 \% " \text { or median lethal dose which is the amount of the } \\
\text { substance required (per bee) to kill } 50 \% \text { of the test population. }\end{array}$ \\
\hline Apis species & Details of Apis bee species. \\
\hline Apis references & $\begin{array}{c}\text { Reference identification number cross-linked to the information provided } \\
\text { in Datasheet } 2 .\end{array}$ \\
\hline $\mathrm{LD}_{50}$ Bombus spp. $\left(\mu \mathrm{g} \mathrm{bee}^{-1}\right)$ & $\begin{array}{l}\text { Lethal Dose, } 50 \% " \text { or median lethal dose which is the amount of the } \\
\text { substance required (per bee) to kill } 50 \% \text { of the test population. }\end{array}$ \\
\hline Bombus species & Details of Bombus bee species. \\
\hline Bombus references & $\begin{array}{c}\text { Reference identification number cross-linked to the information provided } \\
\text { in Datasheet } 2 .\end{array}$ \\
\hline LD $_{50}$ Osmia spp. $\left(\mu \mathrm{g} \mathrm{bee}^{-1}\right)$ & $\begin{array}{l}\text { Lethal Dose, } 50 \% " \text { or median lethal dose which is the amount of the } \\
\text { substance required (per bee) to kill } 50 \% \text { of the test population. }\end{array}$ \\
\hline Osmia species & Details of Osmia bee species. \\
\hline Osmia references & $\begin{array}{l}\text { Reference identification number cross-linked to the information provided } \\
\text { in Datasheet } 2 .\end{array}$ \\
\hline $\mathrm{LD}_{50}$ other bee species $\left(\mu \mathrm{g} \mathrm{bee}^{-1}\right)^{\S}$ & $\begin{array}{l}\text { Lethal Dose, } 50 \% " \text { or median lethal dose which is the amount of the } \\
\text { substance required (per bee) to kill } 50 \% \text { of the test population. }\end{array}$ \\
\hline Other species name & Details of other bee species. \\
\hline Other species references & $\begin{array}{c}\text { Reference identification number cross-linked to the information provided } \\
\text { in Datasheet } 2 .\end{array}$ \\
\hline
\end{tabular}

$\S$ The last three parameters are repeated such that data for two different bee species can be stored for each pesticide/mode of toxicity.

Datasheet 2 provides the reference information for the source of each datum given in the toxicity datasheet cross-referenced by the unique reference number given in datasheet 1 . Datasheet 3 contains user notes.

\subsection{Insights into the Data}

The outcome of this study is a dataset collated from 316 regulatory and peer reviewed articles that contains 178 records covering 120 different pesticides and their variants which includes 142 records for bumblebees and a further 115 records for other wild bee species. General observations of the dataset show that there is still a paucity of useful data for the most commonly used pesticides. Of the 1539 pesticides in the PPDB 382 of these are approved for use in the European Union but only $10.5 \%$ of these has bumblebee data for both topical and dietary exposure, and only $17 \%$ has data for one or the other exposure route.

As mentioned previously pesticide toxicity data for honeybees has until relatively recently been used as a protective surrogate for all bee species and even now, in many instances, this is still the case albeit a safety factor is applied which reduces the honeybee $\mathrm{LD}_{50}$ value by a factor of 10 . As there is continuing debate regarding the validity of using honeybees as a protective species $[17,26]$, it is interesting to consider the statistical correlation between the two species based on the data collated for the dataset described herein. It should, however, be mentioned that this analysis is somewhat crude due to data limitations as the pesticide toxicity values for the two species have not been paired in terms of experimental conditions and worst case $\mathrm{LD}_{50}$ values have been used to account for 'greater than' values. Applying Pearson's correlation between the original topical $\mathrm{LD}_{50}$ values of $A$. mellifera 
and B. terrestris only a weak positive correlation was identified (Pearson's $r=0.423$ for 78 data pairs) implying that honeybee topical toxicity data cannot be used, with confidence, to predict that for bumblebee toxicity. However, investigating the data further it was found that for the majority of the topical acute toxicity data pairs honeybees were indeed equal to or more sensitive to pesticides than bumblebees $(84.6 \%)$ and so using honeybee toxicity values would offer a good level of protection. If the safety factor of 10 is applied the level of protection increases such that in $98.7 \%$ of the data pairs bumblebees would be protected using the adjusted $\mathrm{LD}_{50}$ values for honeybees. In terms of dietary toxicity again a positive correlation was identified but this was even weaker than that for the topical toxicity data values (Pearson's $r=0.305$ for 62 data pairs). For $75.8 \%$ of the data pairs honeybees were more sensitive than bumblebees and this value increases to $95.2 \%$ when the safety factor is applied. Data are much more limited for Osmia species meaning that the analysis is even less reliable, however from the 21 data pairs available for topical toxicity moderate positive correlation (Pearson's $r=0.627$ ) and looking at the data for both topical and dietary data together, Apis mellifera were more sensitive than Osmia spp. in only $73.8 \%$ of the data pairs but this rises to $84.6 \%$ when the safety factor is applied. With the caveat that the analysis here is very crude, it would suggest that the current approach of using honeybees as a surrogate in risk assessments is sound. It also fully supports the reasoning, based on a more comprehensive assessment, albeit based on a smaller dataset, proposed by Thompson and Pamminger [26] that higher tier testing of wild bee species for regulatory purposes is only needed in cases of concern. Nevertheless, it would always be desirable to have quality data for the specific species.

Going forward it is reasonable to assume, due to the inclusion of wild bees in regulatory risk assessments, that the database size will increase opening up opportunities for it to be used in hazard or risk assessment applications, as has been the case with other data in the PPDB. For example, the honeybee data from the PPDB is used to support a Danish pesticide load indicator [27] which aims to encourage more informed decisions relating to pesticide choice. It may also be used in various modelling exercises for evaluating risk as has been done with honeybee data previously. For example the work of Como et al. [28] and Hamadache et al. [29].

Despite the bumblebee dataset only being developed recently it will soon be used within the UK Bee.Watch initiative. This initiative aims to create an efficient means of communication between beekeepers, farmers, pest controllers and the public with the key means of achieving this being a mobile phone 'app' and dedicated website [30]. The initiative provides a means of reporting the collection of honeybee swarms as well as including a process for beekeepers to record bee inspections, colony treatments and losses. A key feature of the app is designed to improve pesticide awareness amongst stakeholders and so facilitate informed decision making. This is done by using it to notify registered users of which pesticide is being used, when and where. Alongside this information is the Bee.Watch hazard indicator system which provides a basic guide to the ecotoxicity of the pesticide active ingredients to a number of different species including honeybees and this will be extended to bumblebees during the second half of 2019. In terms of both honeybees and bumblebees the hazard assessment draws upon two data parameters for each species stored within the PPDB: The topical acute $\mathrm{LD}_{50}$ as $\mu \mathrm{g}$ bee $^{-1}$ and the dietary acute $\mathrm{LD}_{50}$ as $\mu \mathrm{g} \mathrm{bee}^{-1}$. The worst-case value of the two is used to allocate the substance into one of three hazard classes $(1=$ high; $2=$ moderate; and $3=$ low $)$ using the same threshold values applied in many risk assessments [31]. If the value is $>100 \mu \mathrm{g}$ bee $^{-1}$ it falls into class 3; if the value is $<=100$ and $>=1 \mu \mathrm{g}$ bee $^{-1}$ it falls into class 2 ; and if the value is $<1 \mu \mathrm{g}$ bee $^{-1}$ it falls into class 1 . The 3 hazard classes are displayed within Bee.Watch using a traffic light system $(1=$ red; 2 = amber; and 3 = green). When topical or dietary toxicity data are not available, the substance is given an 'unknown' hazard class.

The UK Bee.Watch initiative is quite new, being launched in 2018. The majority of its users are concentrated in Oxfordshire, Berkshire and Buckinghamshire (Figure 1) with pockets of users in Somerset, Lincolnshire, Derbyshire and Shropshire (Figure 1). Users of Bee.Watch are distributed 
between (professional) beekeepers, swarm collectors and coordinators and pesticide users: Both farmers and other professionals.

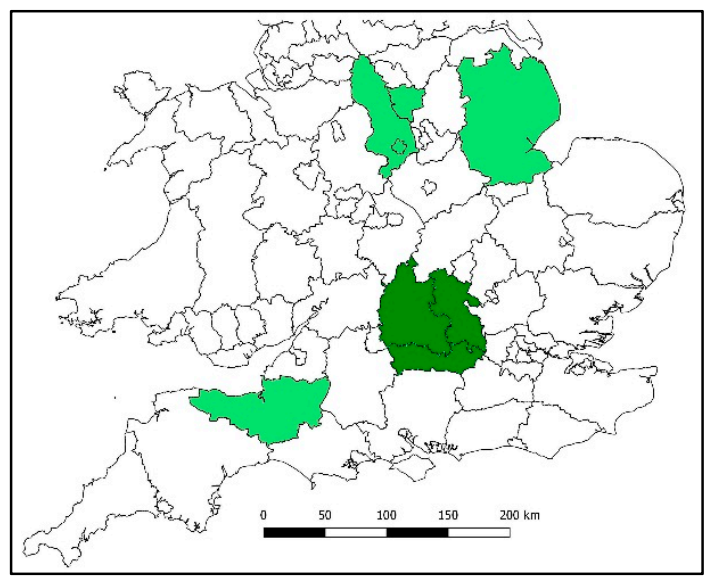

Figure 1. Map depicting the Bee.Watch user-base as of Spring 2019. The dark green area is the main user area. The lighter green areas show the location of small pockets of users.

\section{Methods}

The methodology adopted for this study was two-fold. Firstly, publicly available regulatory dossiers published post-2005 were systematically searched for bumblebee data. As these data have already been peer reviewed as part of the pesticide approvals process, they were accepted as suitable for inclusion without any further quality assessment. For example, dossiers submitted to the European Commission (a process managed by EFSA) as part of Regulation 1107/2009 are peer reviewed by experts from individual member states. Secondly, published articles were identified using an approach similar to that of a systematic review. A review protocol was applied that identified the literature databases to be searched (i.e., Google Scholar, ScienceDirect, Scopus, American Chemical Society Journals Database) and the search procedure to be applied that would identify all (in as far as this is reasonably possible) relevant peer reviewed published literature available during the period January 1970 to June 2019. The review protocol also described various criteria that the study would need to comply with in order for the data to be considered of suitable quality to warrant extraction. These criteria included, for example, evidence that the experimental approach was scientifically sound, that experiments were replicated and that the statistical analysis was appropriate for the study. Only original and empirical data was extracted i.e., that determined experimentally and not modelled or inferred in any way. Other suitable studies were found from the citation lists of the articles identified in the literature searches. Review articles were primarily used to identify suitable studies and not for data extraction. However, in some instances, particularly where the article was old and it was not possible to obtain a copy, data have been used that was quoted in the review article itself. This was not an ideal situation as it had consequences for data quality and so the decision to include data from reviews was based on judgement regarding the value of the data in terms of the amount of other data available on the pesticide/bee species combination.

Once suitable data had been identified they was extracted onto an MS Excel spreadsheet using the structure given in Table 1. Simple software routines were written to assist with data validation and analysis. This included checking for typographical errors and duplicate entries. Where multiple studies were identified addressing the same pesticide/bee species combination and where each met the quality criteria the 'reasonable' worst case value was used, essentially meaning that any outliers were ignored. Regulatory data was given preference to literature sourced data. 


\section{User Notes}

In the dataset, a parameter called 'PPDB Code' is provided for each pesticide active substance. This is a unique number which allows the data in the MS Excel file to be directly associated with the full data profile for that pesticide in the PPDB. This can be accessed by replacing the '\#' with the PPDB Code at the end of the following URL: http://sitem.herts.ac.uk/aeru/ppdb/en/Reports/\#.htm. For example, to access the full dataset for cypermethrin use the URL: http://sitem.herts.ac.uk/aeru/ppdb/en/Reports/197.htm. Alternatively, the PPDB Home page has an A-Z listing as well as a search function [24].

Pesticide active substances are often known by a range of common names and the spelling of these may differ in different languages. If the pesticide required is not found in the dataset, check again using its CAS RN identifier or via an alias. A list of aliases and CAS RN identifiers for that substance can be found in the PPDB and the database search function may be useful in this respect. The full dataset as given in this article's Supplementary file is available in the MS Access and MS excel versions of the database [24].

Future updates will be undertaken periodically using the same approach described herein and added to the PPDB according to its established protocols.

Supplementary Materials: The following are available online at http://www.mdpi.com/2306-5729/4/3/98/s1, Table S1: 'Bee toxicity data June 2019.

Author Contributions: K.A.L. undertook the literature review, evaluation of the studies, collated the raw data and drafted this manuscript. J.T. wrote the analytical routines and subsequently used these to analyse the data, identify duplicates and errors. J.T. was also responsible for production of Figure 1 and the formatting of the final dataset presented with this manuscript as well as proofreading the manuscript. Both authors agree to be listed and have approved the submitted article.

Funding: This research received no direct external funding. It was undertaken as part of the PPDB updating process funded by PPDB licensing and consultancy fees.

Acknowledgments: The authors express their gratitude to the PPDB user base for their continued support. The support of colleagues within the Agriculture and Environment Research Unit (AERU) at the University of Hertfordshire and those from Bee.Watch are gratefully acknowledged.

Conflicts of Interest: The authors declare no conflict of interest.

\section{Abbreviations}

The following abbreviations are used in this manuscript:

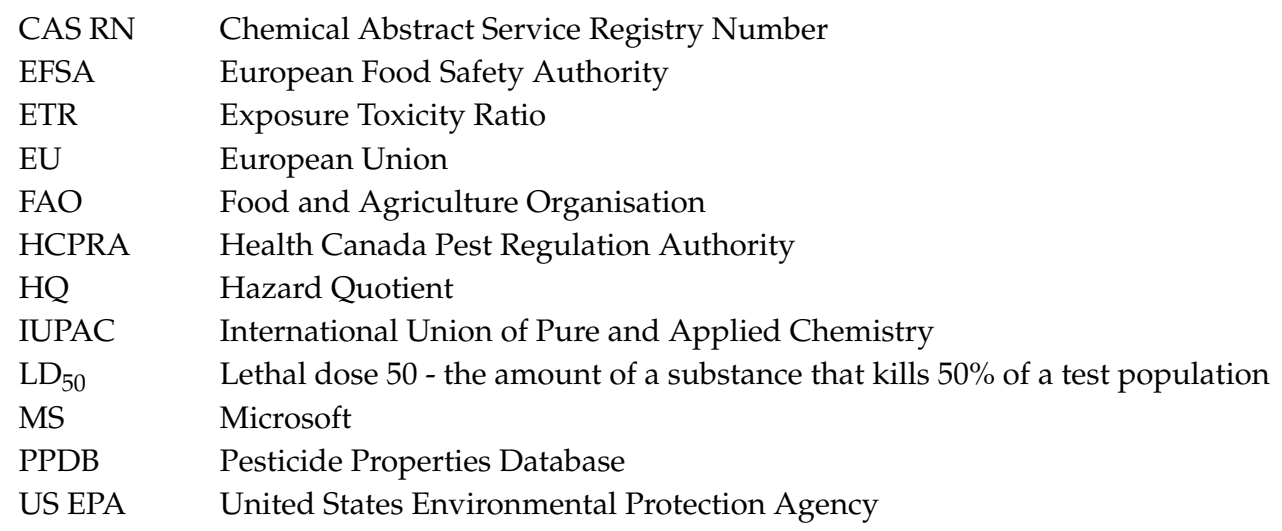

\section{References}

1. Williams, I.H. Insect Pollination and Crop Production: A European Perspective. Pollinating Bees-The Conservation Link between Agriculture and Nature; Ministry of Environment: Brasilia, Brazil, 2002; pp. 59-65.

2. Klein, A.-M.; Vaissiere, B.E.; Cane, J.H.; Steffan-Dewenter, I.; Cunningham, S.A.; Kremen, C.; Tscharntke, T. Importance of pollinators in changing landscapes for world crops. Proc. R. Soc. B Biol. Sci. 2007, 274, 303-313. [CrossRef]

3. Carreck, N.; Williams, I. The economic value of bees in the UK. Bee World 1998, 79, 115-123. [CrossRef] 
4. Goulson, D.; Lye, G.C.; Darvill, B. Decline and conservation of bumble bees. Annu. Rev. Entomol. 2008, 53, 191-208. [CrossRef]

5. Russell, A.L.; Leonard, A.S.; Gillette, H.D.; Papaj, D.R. Concealed floral rewards and the role of experience in floral sonication by bees. Anim. Behav. 2016, 120, 83-91. [CrossRef]

6. Gallai, N.; Salles, J.M.; Settele, J.; Vaissière, B.E. Economic valuation of the vulnerability of world agriculture confronted with pollinator decline. Ecol. Econ. 2009, 68, 810-821. [CrossRef]

7. Buglife. Pollination. 2019. Available online: https://www.buglife.org.uk/bugs-and-habitats/pollination (accessed on 17 June 2019).

8. Parliamentary Office of Science and Technology-POST. Insect Pollination, PostNote Number 348, January 2010. Available online: https://www.parliament.uk/documents/post/postpn348.pdf (accessed on 17 June 2019).

9. Smith, P.; Ashmore, M.; Black, H.; Burgess, P.; Evans, C.; Hails, R.; Potts, S.; Quine, T.; Thomson, A.; Biesmeijer, K.; et al. Regulating Services, in The U.K National Ecosystem Assessment Technical Report: U.K National Ecosystem Assessment; UNEP-WCMC: Cambridge, UK, 2011; pp. 535-597.

10. Rortais, A.; Arnold, G.; Dorne, J.L.; More, S.J.; Sperandio, G.; Streissl, F.; Szentes, C.; Verdonck, F. Risk assessment of pesticides and other stressors in bees: Principles, data gaps and perspectives from the European Food Safety Authority. Sci. Total Environ. 2017, 587, 524-537. [CrossRef]

11. Gradish, A.E.; Van Der Steen, J.; Scott-Dupree, C.D.; Cabrera, A.R.; Cutler, G.C.; Goulson, D.; Klein, O.; Lehmann, D.M.; Lückmann, J.; O’Neill, B.; et al. Comparison of Pesticide Exposure in Honey Bees (Hymenoptera: Apidae) and BumbleBees (Hymenoptera: Apidae): Implications for Risk Assessments. Environ. Entom. 2018, 48, 12-21. [CrossRef]

12. Thompson, H. Extrapolation of acute toxicity across bee species. Integr. Environ. Assess. Manag. 2016, 12, 622-626. [CrossRef]

13. European Food Safety Authority-EFSA. Guidance on the risk assessment of plant protection products on bees (Apis mellifera, Bombus spp. and solitary bees). EFSA J. 2013, 11, 3295.

14. US Environmental Protection Agency-US EPA; Health Canada; California Department of Pesticide Regulation. Guidance for Assessing Pesticide Risk to Bees. June 2014. Available online: file://D:/bee\%20paper/Pollinator\%20toxicity/Background\%20references/pollinator_risk_assessment_ guidance_06_19_14.pdf (accessed on 17 June 2019).

15. Thompson, H.M. Assessing the exposure and toxicity of pesticides to bumblebees (Bombus sp.). Apidologie 2001, 32, 305-321. [CrossRef]

16. Arena, M.; Sgolastra, F. A meta-analysis comparing the sensitivity of bees to pesticides. Ecotox 2014, 23, 324-334. [CrossRef]

17. Uhl, P.; Awanbor, O.; Schulz, R.S.; Brühl, C.A. Osmia bicornis is rarely an adequate regulatory surrogate species. Comparing its acute sensitivity towards multiple insecticides with regulatory Apis mellifera endpoints. BioRxiv 2018, 366237. [CrossRef]

18. Kovács-Hostyánszki, A.; Földesi, R.; Báldi, A.; Endrédi, A.; Jordán, F. The vulnerability of plant-pollinator communities to honeybee decline: A comparative network analysis in different habitat types. Ecol. Ind. 2019, 97, 35-50. [CrossRef]

19. Banks, J.E.; Stark, J.D.; Vargas, R.I.; Ackleh, A.S. Deconstructing the surrogate species concept: A life history approach to the protection of ecosystem services. Ecol. Appl. 2014, 24, 770-778. [CrossRef]

20. Yue, M.; Luo, S.; Liu, J.; Wu, J. Apis cerana is less sensitive to most neonicotinoids, despite of their smaller body mass. J. Econ. Entomol. 2017, 111, 39-42. [CrossRef]

21. Turner, J.A. The pesticide manual: A world compendium, 18th ed.; British Crop Production Council: East Hampshire, UK, 2018.

22. Oregon State University; U.S. Environmental Protection Agency; National Pesticide Information Centre-NPIC. Available online: http://www.npic.orst.edu/ (accessed on 17 June 2019).

23. Kegley, S.E.; Hill, B.R.; Orme, S.; Choi, A.H. PAN Pesticide Database. Pesticide Action Network, North America (San.) 2000. Available online: http://pesticideinfo.org/ (accessed on 11 July 2019).

24. Lewis, K.A.; Tzilivakis, J.; Warner, D.J.; Green, A. An international database for pesticide risk assessments and management. Hum. Ecol. Risk Assess. 2016, 22, 1050-1064. [CrossRef]

25. Sanchez-Bayo, F.; Goka, K. Pesticide residues and bees-a risk assessment. PLoS ONE 2014, 9, e94482. [CrossRef] 
26. Thompson, H.M.; Pamminger, T. Are honeybees suitable surrogates for use in pesticide risk assessments for non-Apis bees? Pest. Manag. Sci. 2019. [CrossRef]

27. Kudsk, P.; Jørgensen, L.N.; Ørum, J.E. Pesticide Load-A new Danish pesticide risk indicator with multiple applications. Land Use Pol. 2018, 70, 384-393. [CrossRef]

28. Como, F.; Carnesecchi, E.; Volani, S.; Dorne, J.L.; Richardson, J.; Bassan, A.; Pavan, M.; Benfenati, E. Predicting acute contact toxicity of pesticides in honeybees (Apis mellifera) through a k-nearest neighbor model. Chemosphere 2017, 166, 438-444. [CrossRef]

29. Hamadache, M.; Benkortbi, O.; Hanini, S.; Amrane, A. QSAR modeling in ecotoxicological risk assessment: Application to the prediction of acute contact toxicity of pesticides on bees (Apis mellifera L.). Environ. Sci. Poll. Res. 2018, 25, 896-907. [CrossRef]

30. Bee.Watch. Keeping Bees on Things. Available online: https://www.bee.watch/ (accessed on 17 June 2019).

31. Medrzycki, P.; Giffard, H.; Aupinel, P.; Belzunces, L.P.; Chauzat, M.P.; Classen, C.; Colin, M.E.; Dupont, T.; Girolami, V.; Johnson, R.; et al. Standard methods for toxicology research in Apis mellifera. J. Apicult. Res. 2013, 52, 1-60. [CrossRef]

(C) 2019 by the authors. Licensee MDPI, Basel, Switzerland. This article is an open access article distributed under the terms and conditions of the Creative Commons Attribution (CC BY) license (http://creativecommons.org/licenses/by/4.0/). 